Théologiques

Théologiques

\title{
Canonicité et philosophie herméneutique
}

\section{Jean Grondin}

Volume 1, numéro 2, octobre 1993

Les textes fondateurs. Entre autorité et liberté

URI : https://id.erudit.org/iderudit/602388ar

DOI : https://doi.org/10.7202/602388ar

Aller au sommaire du numéro

Éditeur(s)

Faculté de théologie de l'Université de Montréal

ISSN

1188-7109 (imprimé)

1492-1413 (numérique)

Découvrir la revue

Citer cet article

Grondin, J. (1993). Canonicité et philosophie herméneutique. Théologiques, 1(2), 9-23. https://doi.org/10.7202/602388ar

\section{Résumé de l'article}

Le propos de cet article est de faire ressortir l'actualité que peut avoir la notion de canonicité dans la perspective d'une philosophie herméneutique. Si l'on peut dire de la philosophie moderne qu'elle est en général hostile à toute autorité qui viendrait s'imposer de l'extérieur à la raison, la mise en question, par l'herméneutique, de la souveraineté de la subjectivité pourrait insuffler un nouveau sens à l'idée de canonicité. La secondarité de la conscience en regard d'un canon qui la précède décrit en effet un phénomène qui se retrouve dans tous les savoirs. Ce qui distingue la théologie, c'est peut-être seulement qu'elle en ait une conscience explicite. 


\title{
Canonicité ef philosophie herméneutique.
}

\author{
Jean GRONDIN \\ Département de philosophie \\ Université de Montréal
}

\section{RÉSUMÉ}

Le propos de cet article est de faire ressortir l'actualité que peut avoir la notion de canonicité dans la perspective d'une philosophie herméneutique. Si l'on peut dire de la philosophie moderne qu'elle est en général hostile à toute autorité qui viendrait s'imposer de l'extérieur à la raison, la mise en question, par l'herméneutique, de la souveraineté de la subjectivité pourrait insuffler un nouveau sens à l'idée de canonicité. La secondarité de la conscience en regard d'un canon qui la précède décrit en effet un phénomène qui se retrouve dans tous les savoirs. Ce qui distingue la théologie, c'est peut-être seulement qu'elle en ait une conscience explicite.

Depuis au moins deux siècles, philosophie et canonicité ne font pas bon ménage, c'est-à-dire, assez généralement, depuis la prise de conscience de soi de la modernité que représente Kant. Répondant luimême à l'exhortation de Hume, Kant a incité sa postérité à se défaire de toute autorité extérieure à la raison et à rejeter tout le "canon" de la métaphysique traditionnelle, taxée d'illusion puisqu'elle prétendait offrir une connaissance d'un monde transcendant. C'était radicaliser l'exigence cartésienne d'une philosophie qui s'en tienne à ce qui est immédiatement évident à la conscience du sujet pensant. Le seul canon que Kant reconnaîtra lui-même sera donc le "Canon de la raison pure", selon 
l'intitulé de ce qui constitue à toutes fins utiles la conclusion de sa Critique de 1781 1. La raison - celle de la subjectivité bien sûr - ne reconnaît plus d'autre canon que celui qu'elle peut produire à partir d'elle-même, "dans les limites de la simple raison", suivant cette fois le titre du livre de 1793 sur la Religion. La raison devient la seule autorité philosophique ou scientifique et les normes de son usage, son "canon", seront arrêtées par la raison elle-même. Kant porte ainsi à son terme la devise des Lumières, "aie le courage de te servir de ton propre entendement!" 2. II demeure que Kant aura peut-être été l'un des derniers philosophes à reconnaître encore une quelconque dignité, une très grande en fait, à la notion de canon. Mais, en toute logique, si la raison est capable de penser de façon autonome, elle n'a plus vraiment besoin d'un canon ${ }^{3}$. La notion de canon évoque en effet l'idée d'une autorité qui se trouverait comme imposée de l'extérieur à l'entendement. Depuis Kant, et dans ce qui est certainement l'une des suites conséquentes de sa modernité, la philosophie, s'auto-définissant par l'exercice libre et autonome de la réflexion critique, a donc voulu s'affranchir de toute autorité canonique. Il est inutile d'ajouter que ceci est aussi vrai de la civilisation contemporaine en général, transie qu'elle est, quoi qu'on en dise, des exigences de la métaphysique de la subjectivité et de son exaltation de l'individualité prétendument autonome. Après Kant, la métaphysique de la subjectivité s'est en tout cas si bien implantée que la notion de canon n'est presque plus jamais réapparue en philosophie.

L'imposition d'un canon à la pensée équivaut dorénavant à une paresse de la raison. L'opposition de la philosophie et de la canonicité incarne donc une des formes de la confrontation entre la Modernité et l'Antiquité, la rationalité et la tradition, les lumières et l'obscurité, et où c'est toujours une rationalité sûre d'elle-même qui dessine la caricature des temps qu'elle proclame avoir dépassés. Les notions d'“Antiquité" et de "Moyen" Âge qui seront créées à cette fin caractériseront avant tout une absence de matûrité en regard des Temps Modernes, les premiers à être éclairés, ou à se prétendre plus clainoyants que les autres. Cette modernité se manifestera aussi dans

\footnotetext{
1 Voir à ce propos notre étude «La conclusion de la Critique de la raison pure», Kant. Studien 81 (1990) 129-144.

2 Voir E. KANT, «Réponse à la question: Qu'est-ce que les Lumières?» (1785), dans Oeuvres philosophiques. t. II. (Pléiade), Paris, Gallimard, 1986, 207.

3 Ceci aura peut-être conduit les interprètes à négliger, à tort, le Canon que Kant pensait encore devoir assigner à la raison, fût-il auto-imposé.
} 
l'attitude nouvelle qui prévaudra au sein même des recherches théologiques sur le canon des Écritures. En période de théologie "libérale", ainsi nommée parce qu'elle résulte justement d'une "libération" en regard d'une canonicité étroite, les études historiques et systématiques sur les écrits canoniques tendront à mettre en évidence le caractère accidentel et fortuit de ce qui s'est constitué comme ensemble d'écrits canoniques. Ce sont des décisions historiques, situables et relativisables, qui auraient constitué le canon. Un canon dont la genèse est arbitraire perd nécessairement de son autorité. Le canon apparaît de plus en plus comme un legs, sinon, en exagérant à peine, un embarras historique en regard duquel une discipline traditionnellement aussi "canonoïde" que la théologie peut cultiver une certaine distance critique. C'est l'intention philosophique, disons kantienne tant la descendance est directe, de la "méthode" historico-critique - qu'on ne peut "critiquer" à son tour sans s'exclure de la modernité. Après Kant, et en attendant une réhabilitation "barthienne", mais souvent gratuite, de la notion de révélation, la raison critique prendra le pas sur l'autorité du canon ${ }^{4}$. Cette même raison sera dès lors habilitée à déceler des contradictions théologiques irréconciliables, et pas seulement des incohérences historiques, dans le corps des Écritures. Devant ces contradictions - et il n'y a pas de principe plus rationnel que celui de contradiction -, la raison devra "choisir" les doctrines qu'elle jugera plus essentielles 5.

Si la notion de canonicité bénéficie aujourd'hui d'une nouvelle actualité, c'est peut-être encore une fois pour des raisons philosophiques. C'est que les assises de la philosophie moderne de la subjectivité, qu'elle soit cartésienne, kantienne ou encore nietzschéenne, ont été elles-mêmes mises en question. Pour le dire tautologiquement, il est devenu de plus en plus clair que la métaphysique de la subjectivité était elle-même une métaphysique, c'est-à-dire qu'elle comportait des présupposés qui ne résistent pas à l'examen d'une rationalité qui apprend à être critique d'elle-même. On a parlé de

${ }^{4}$ Cf. à titre exemplaire l'écrit de jeunesse de J. G. FICHTE, Essai d'une critique de toute révélation (1792). Paris, Vrin, 1988.

5 C'est la thèse courageuse de P. LAMARCHE, «Hypothèses à propos des divergences théologiques dans le Nouveau Testament», dans Le Canon des Écritures. Etudes historiques, exégétiques et systématiques. (THEOBALD, C. ,éd.), Paris, Cerf, 1990, 441-491. Dans le même recueil, B. SESBOUÉ («Essai de théologie systématique sur le canon des Écritures", 523-539, surtout 537 au sujet du "postulat de l'unité de sens du canon") cherche plutôt à atténuer ce type de contradictions (sans toutefois viser directement P. LAMARCHE, mais l'opposition apparaît assez frontale). 
"postmodernisme" pour caractériser cette époque où la modernité en vient à douter de sa propre autorité. Depuis Hegel - mais qui le proclamait encore pour célébrer la rationalité de l'histoire - la raison s'avise douloureusement qu'elle possède aussi une histoire. Ce qui est clair et distinct pour une subjectivité du XVIlle siècle ne le sera peutêtre plus pour celle du XXe, dont les positivités, les nôtres, seront soumises à la même destruction par les générations futures. La raison n'est pas elle-même exempte de contingence ${ }^{6}$. Alors qu'elle s'estimait être la source de toute intelligibilité, la raison se découvre projetée dans des horizons préalables de sens dont elle ne maîtrise plus tous les ressorts. La subjectivité s'inscrit toujours déjà dans des contextes d'interprétation, dans des traditions donc, même là où elle s'affiche superbement autonome. Cet âge où tout n'est "qu'interprétation" est celui de l'herméneutique?

Néanmoins, c'est peut-être succomber à une certaine facilité que d'apercevoir un postmodernisme dans cette nouvelle constellation de l'esprit du temps. C'est que l'herméneutique reste, en un sens précieux, un accomplissement de la modernité. Parvenue à l'herméneutique, la subjectivité est seulement devenue critique à l'endroit de ses propres contenus. Elle apprend à voir que ses évidences ne sont pas nécessairement l'a priori de toute vérité, ces évidences étant peut-être elles-mêmes le fait d'interprétations qui ont aussi leur histoire. L'herméneutique est donc plus "critique", plus moderne donc, que la modernité qui ne s'interrogeait pas (encore) sur les présupposés historiques de la subjectivité. Le sujet connaissant ou agissant se meut au sein de préjugés qui le précèdent et qui l'orientent. L'important, selon l'herméneutique, n'est pas de sortir du cercle des préjugés, comme le croyaient naïvement les Lumières 8 , mais d'en prendre conscience, afin justement de soumettre nos anticipations historiques à un examen critique qui demeure essentiellement ouvert. L'âge de l'herméneutique est ainsi celui d'une perpétuelle remise en question de soi, d'un débat infini de la vérité avec elle-même.

6 La notion de contingence deviendra dès lors l'un des maîtres mots de la philosophie postmoderne. Cf. tout spécialement R. RORTY, Contingency, Irony, and Solidarity. Cambridge, Cambridge University Press, 1989.

${ }^{7}$ Cf. notre étude sur L'universalité de l'herméneutique. Paris, PUF, 1993.

8 Cf. Hans-Georg GADAMER, Wahrheit und Methode. Tübingen, Mohr, 1960; Vérité et méthode. Paris, Seuil, 1976. 
C'est dans ce contexte que la notion de canonicité acquiert une nouvelle pertinence. La subjectivité, en effet, ne s'oppose plus à la tradition ou à une autorité jugée canonique, elle se sait elle-même redevable d'un horizon de sens, d'une hiérarchie d'évidences qu'on peut bien qualifier de canoniques. Ces évidences qui forment la base de nos jugements et de nos appréciations de la vérité, viennent généralement des influences que nous avons reçues et dont la recomposition, à chaque fois unique, organise l'unité de notre personnalité. Être poreux, l'homme ne cesse jamais de s'éduquer et de s'orienter en fonction de points de repère canoniques, canonicité qui répond secrètement à la désorientation d'un être projeté dans une existence dont it ne maîtrise pas la contingence. On a beau insister, avec toute la démagogie du monde, sur la créativité et l'imagination en pédagogie, il demeure que l'éducation est d'abord quelque chose que l'on reçoit, depuis la plus tendre enfance d'ailleurs, et qu'elle se fait par l'assimilation critique de balises canoniques (la grammaire, l'histoire, les modes de vie, etc.). Cette éducation, qu'on appelle aussi "formation" tant elle façonne notre être, nous procure, sans que l'on en ait toujours conscience, un fonds de valeurs et de références qui méritent d'être appelées canoniques parce qu'elles font autorité.

Cela est assez connu. Ce qui est un peu plus nouveau, c'est que les canons qui s'offrent ainsi à notre formation soient devenus aussi pluriels. II n'y a plus une seule formation "canonique". Le cercle des vérités canoniques se détermine lui-même en fonction de lectures du réel qui peuvent être conflictuelles. Cette pluralité des canonicités, renforcée sans doute par la tendance mondiale vers la globalisation et l'amalgame des cultures, se trouve à la racine d'un des malaises les plus profonds de notre système d'éducation, celui du manque de formation dite fondamentale. Quelles sont les oeuvres qu'il faut avoir assimilées pour jouir d'une culture de base dans notre société? Homère, Shakespeare, Mozart, Kafka? Mais déjà l'idée de privilégier quelques chefs-d'oeuvre accrédités par la tradition peut elle-même être taxée d'idéologie. Ne faudrait-il pas faire une plus grande place dans le "canon" des classiques aux traditions plus nationales, aux minorités opprimées, etc. On sait à quel type de conflits et de suspicions peut conduire cette volonté d'instituer un canon commun (ce que les Américains appellent un core curriculum) là où la désorientation générale est devenue pathologique ${ }^{9}$. Pareille institution sera toujours

9 C'est à cette désorientation qu'ont voulu réagir les livres de E. D. HIRSCH, Cultural Literacy: What every American needs to know. Boston, Houghton Mifflin, 1987 et A. 
soupçonnée d'arbitraire et de parti pris. Ce problème qui touche toutes les disciplines a en quelque sorte l'avantage d'être déjà "résolu" en théologie. Elle est peut-être la seule discipline qui n'ait pas à s'interroger sur la constitution de son propre canon. Ce canon existe déjà. Cela ne le rend pas moins contingent pour autant. La théologie ne le consteste pas, admettant volontiers qu'il résulte de décisions historiques. Tout ce qui est pluriel en théologie, c'est l'interprétation du canon, ce n'est pas le canon lui-même. Cette pluralité elle-même n'est pas une catastrophe puisque les questions de la théologie n'existent que dans la mesure et l'espace où elles sont débattues.

Tous les savoirs dépendent à un degré ou l'autre d'un fonds de connaissance préalable qui n'est pas systématiquement remis en cause. Dans la mesure où l'on n'a pas affaire à un endoctrinement chronique, il est aussi normal qu'il en soit ainsi. Puisque personne n'a le temps de tout remettre en question et de confronter toutes les interprétations concurrentes, il faut s'en remettre, et moins paresseusement qu'on ne le croit, à l'autorité des références canoniques qui ont été transmises. Thomas S. Kuhn a montré l'influence souterraine qu'exerçaient les manuels ou les text-books sur des générations de scientifiques 10 . On chantera tant qu'on voudra les vertus de l'expérience directe dans la formation scientifique, cette formation continue de provenir pour une très large part des maîtres et des text-books, c'est-à dire de vérités reçues ou simplement lues. La théorie des paradigmes de Kuhn a connu trop de retentissements dans la théologie des trois dernières décennies pour qu'il soit nécessaire d'y insister. La seule chose qui surprenne en fait, c'est que les théologiens aient cru avoir quelque chose à apprendre de Kuhn. En effet, la théologie qui s'est inspirée de Kuhn n'a pas vu que c'était plutôt Kuhn qui suivait la logique canonique de la théologie. La théologie sait depuis longtemps, sinon depuis toujours, qu'elle est liée à un canon qui la précède et qui gouverne la vérité de ses énoncés même si ses origines sont plus ou moins contingentes ou relativisables. La théologie connaît aussi les abus de l'autorité qui se prétend canonique et les avantages qu'il peut y avoir à manier plus libéralement le canon et toutes les formes d'autorité dont l'Église possède une incomparable expérience. On pourrait même dire que la théologie, idéalement, est la

BLOOM, The Closing of the American Mind. New York, Simon \& Schuster, 1987 qui ont suscité une très vaste discussion dans les médias américains.

10 Th. S. KUHN, The Structure of Scientific Revolutions. Chicago, Chicago University Press, 1962, 2e édition 1970; tr. franç. La structure des révolutions scientifiques. Paris, Flammarion-Champs, 1983. 
Jean Grondin: Canonicité et philosophie...

moins "dogmatique" des sciences dans le sens où elle se sait expressément rivée à un canon ${ }^{11}$. Elle élève ainsi à la conscience une dépendance en regard de l'autorité que les autres sciences admettent beaucoup moins volontiers, en commençant par la philosophie qui est pourtant aussi constituée par une tradition de textes fondateurs. Dans les sciences autres que la théologie et la philosophie, ces textes fondateurs (qui peuvent n'être que des text-books ou des notes de cours) ont le malheur d'être moins en évidence. La canonicité ne refléterait donc pas un retard pour la théologie, mais bien son avance vis-à-vis des autres sciences dans la mesure, bien évidemment, où elle sait s'accompagner d'une réflexivité critique à propos de la contingence de sa propre rationalité. Cette réflexivité herméneutique manque du tout au tout dans les disciplines qui se déclarent souveraines en regard de l'autorité de leurs "textes" fondateurs. La théologie pourrait donc être - en ce sens, puisqu'elle l'était déjà au plan de sa pratique exégétique la plus herméneutique de toutes les sciences. Elle est aussi la plus consciemment kuhnienne de toutes, celle qui a toujours su sa dépendance en regard d'un canon, contingent par bien des côtés, mais qui n'en est pas moins condition de vérité. C'est donc à tort qu'on parlera d'une application des idées de Kuhn à la théologie. Historiquement, il est plus juste de reconnaître que Kuhn n'a fait qu'appliquer une vérité théologique aux autres sciences.

On peut donc parler ici d'une fonction paradigmatique de la théologie en regard des autres sciences 12. La limite de la théologie vient, bien entendu, de ce qu'elle est arrimée à un canon historique particulier (limite qu'elle partage toutefois avec bien d'autres disciplines). Ce n'est donc pas à elle qu'il revient de penser les conditions de la canonicité en général. Cette tâche universelle pourrait peut-être revenir à la philosophie. Cette dernière est justement, en ces temps posthégéliens, en mal de catégories universelles. À notre connaissance cependant, il n'y

${ }^{11}$ Mes amis théologiens voudront bien pardonner le caractère peut-être un peu provoquant de cette idéalisation typiquement philosophique. Mais je cherche moins à affoler les théologiens, qui trouveront déjà assez de provocations dans leurs textes canoniques ( $l$ Co $1,23, G a 5,11$, etc.), que les scientifiques et les philosophes qui se professent affranchis de toute positivité. Il est clair que le magistère abuse assez souvent de cette fonction canonique qu'il prétend contrôler. Se privant d'herméneutique, le magistère méconnaît alors que le canon doit toujours faire l'objet d'une reconnaissance si son autorité doit être acceptée.

12 On comparera à ce sujet les remarques de J. SIMON, «Zur philosophischen Ortsbestimmung theologischer Wissenschaft von ihrem Gegenstand her», Tübinger Theologische Quartalschrift 157 (1977) 204-207. 
a encore que très peu d'études philosophiques sur la notion de canonicité $e^{13}$. Peut-être la jugeait-on trop provinciale, limitée qu'elle était à des disciplines aussi "autoritaires" que la théologie et le droit. On a vu que l'on pouvait même parler d'un rejet radical de la canonicité pour la modernité. II se pourrait cependant que le crépuscule de la métaphysique de la subjectivité confère une certaine universalité à l'idée de canonicité ${ }^{14}$. Elle dénoterait la nécessaire secondarité de la raison en regard d'ouvertures de sens qui la devancent, bien qu'elles restent toujours révisables à la faveur de découvertes ou de prises de conscience dont nul ne peut prédire la logique.

Mais reconnaître la dette de la pensée vis-à-vis d'un "canon", n'est-ce pas avaliser une nouvelle paresse de la pensée, une antiAufklärung qui sanctionnerait dogmatiquement l'incapacité de se servir de son propre entendement? Non, c'est assumer jusqu'au bout la finitude de la raison découverte par les Lumières lorsqu'elles ont voulu cesser de mesurer les réalisations humaines à l'aune d'une rationalité infinie. Prendre acte de cette rationalité finie, c'est reconnaître ce qu'on pourrait appeler la temporalité radicale de la pensée humaine, son inscription dans une dialogique historique de la question et de la réponse. Le savoir ne s'institue jamais à un point zéro de l'histoire ou du temps. Penser (connaître, savoir, interpréter, aimer, etc.), c'est tenter de

13 La plupart viennent d'ailleurs d'auteurs proches de l'herméneutique. Cf. notamment T. GRIFFERO, «Ermeneutica e canonicità dei testi», Rivista di estetica, année XXVI (1985) $n^{\circ} 19-20,93-111$ (surtout 108 ss.); L. STERN, «On Interpreting», Journal of Aesthetics and Art Criticism (1980) 119-129; C. ARTHUR, "Gadamer and Hirsch: The Canonical Work and the Interpreter's Intention», Cultural Hermeneutics 4 (1977) 183 197; F. MARTY, «Le Canon des Écritures: Mémoire pour un avenir», dans Le Canon des Écritures. Études historiques, exégétiques et systématiques. (THEOBALD, C., éd.), Paris, Cerf, 1990, 495-512. On pensera aussi aux chapitres que GADAMER a consacrés à la distance temporelle et à la notion de classique dans Vérité et méthode, mais sans aborder thématiquement le problème de la canonicité. Il faut aussi mentionner l'étude, issue de l'école de Dilthey, d'Erich ROTHACKER, «Der Begriff der dogmatischen Denkform», Abhandlungen der Akademie der Wissenschaften und Literatur in Mainz 6 (1954) 11-26. Signe de l'importance qu'il lui prête, GADAMER l'a reprise dans le recueil des grands classiques de l'histoire de l'herméneutique qu'il a édité avec Gottfried BOEHM, Seminar: Philosophische Hermeneutik. Frankfurt am Main, Suhrkamp, 1976, 221-238, où on ne l'aurait pas nécessairement attendue. Sur la notion de "secondarité" qu'on emploiera ici, cf. aussi R. BRAGUE, Europe, la voie romaine. Paris, Criterion, 1992.

14 On en trouve un écho en théologie dans l'intronisation de la nouvelle discipline du "canonical criticism". Cf. à ce sujet les travaux de James A. SANDERS, Identité de la Bible. Torah et Canon. Paris, Cerf, 1975 et Canon and Community. A Guide to Canonical Criticism. (TUCKER, Gene M. , ed.), Philadelphia, Fortress Press, 1984, 
Jean Grondin: Canonicité et philosophie...

répondre à une question qui nous précède, c'est aussi poser une question à la réponse qui était là avant nous et dont nous ne maîtrisons pas tous les termes. Tout effort de pensée est redevable d'une ouverture de sens et dont la canonicité n'est que l'illustration la plus objective. La pensée ne souffre - de naïveté notamment - que de l'ignorer.

La secondarité canonique de la pensée plonge ses racines dans l'expérience du langage 15. Nul n'a créé de toutes pièces le langage (quotidien, rituel ou scientifique) qu'il utilise. Notre capacité de parler provient d'une communauté de langue, de nos proches et de ceux qui nous ont appris à parler pour dire ce qui nous encercle. Savoir parler, c'est avoir appris le langage des autres avec assez d'adresse pour pouvoir être en retour compris par eux. Sans toujours le savoir, parce qu'il nous faudrait à chaque fois un méta-langage pour comprendre ce que nous proférons nous-mêmes, nous nous approprions en même temps la charge émotive que les mots ont acquise et qui fait en sorte, par exemple, que telle expression soit à la mode, telle autre recherchée, déplacée, etc. Grandir dans un langage, c'est apprendre à habiter, à pratiquer un canon linguistique. Aucune signification ne nous appartient en propre, comme l'a rappelé Wittgenstein avec ses fameuses thèses sur le langage privé qui voulaient faire pièce à la conception picturale du langage. Une signification $n^{\prime} a$ de sens que si elle est partagée. C'est la condition essentielle de la compréhension mutuelle qui résulte de l'héritage historique d'une langue donnée. En ce sens, on peut dire que le langage est le premier des canons pour une pensée de la canonicité. La tabula rasa, le degré zéro du savoir qui contemplerait le "réel" dans toute sa nudité, devrait commencer par faire table rase du langage lui-même qui dit ce réel, qui l'habille toujours déjà. Vouloir recommencer à zéro, ce serait faire comme si le langage, et l'expérience qu'il charrie, n'existait pas ou pouvait être reconstruit à neuf. L'utopie de la tabula rasa décrit une situation dans laquelle il serait impossible de savoir quoi que ce soit.

La "canonicité" du langage nous a donc déjà devancés. Temporellement et géographiquement, elle était là avant nous, "là", c'est-à-dire dans la clairière d'intelligibilité que nous habitons lorsque nous parlons. II n'est pas nécessaire que cette dette en regard d'un canon de sens (qui n'est pas d'abord celui des dictionnaires!) soit expressément reconnue. Elle va toujours de soi et, de fait, il nous est tout à fait impossible de rendre pleinement compte de ce que nous disons. Toute

15 Pour ce qui suit, voir notre étude sur «L'intelligence herméneutique du langage», dans L'horizon herméneutique de la pensée contemporaine. Paris, Vrin, 1993. 
signification renvoie à une autre, laquelle enclenche à son tour une infinité virtuelle de renvois. Cette intuition remonte à Leibniz. Selon lui, seul Dieu possède une connaissance distincte et logiquement adéquate de ses propres concepts, c'est-à-dire qui soit capable d'énumérer la totalité des "marques" que renferment ses notions. Les hommes ne peuvent jamais espérer une telle clarté ultime, mais seulement une clarté "esthétique", celle des images plus ou moins vagues, mais qui suffit pour distinguer les choses les unes des autres 16 . II ne nous est pas possible d'expliquer de manière définitive ce que signifient les concepts que nous employons. Pour y parvenir, il faudrait aussi pouvoir expliquer le sens des concepts à l'aide desquels nous élucidons nos propres concepts. On constatera à l'aide de n'importe quel exemple que la pensée humaine se trouve ici condamnée à une régression infinie et que seul Dieu - ou le Dieu logique de la métaphysique - est en mesure de parcourir. Nous ne maîtrisons en réalité aucune signification car nous sommes incapables d'en satisfaire les réquisits logiques d'explication. Aux explications infinies nous substituons - "pour abréger", ironise Leibniz - des signes qui fonctionnent comme des références à des ententes canoniques préalables, comme si les définitions exhaustives étaient déjà en notre possession ${ }^{17}$. Dieu merci, la clarté logique n'est pas nécessaire à la compréhension mutuelle. Les significations qui donnent sens à notre monde n'ont pas à être maîtrisées, mais seulement partagées. Pour se comprendre, il n'est pas nécessaire de s'entendre au préalable sur des définitions de concepts - cela est rigoureusement impossible -, il faut seulement pouvoir s'appuyer sur un fonds commun de sens et dont l"autorité" unit ceux et celles qui parlent. Sans un tel

16 Cf. G.W. LEIBNIZ, «Méditations sur la connaissance, la vérité et les idées (1684)», dans Opuscules philosophiques choisis. Paris, Vrin, 1969, 9-16, et les Nouveaux essais sur l'entendement humain (1703), livre II, chapitre XXIX. Sur l'importance de cette intuition leibnizienne pour la philosophie du langage, cf. J. SIMON, Sprachphilosophie. München / Freiburg, Karl Alber Verlag, 1981. La dérive infinie des signes les uns vers les autres, bien vue par Leibniz, préfigure ce que Jacques DERRIDA (De la grammatologie. Paris, Minuit, 1968) a appelé la différance du sens. Quelques indications à ce propos dans F. MARTY, op. cit., 497.

17 Cf. G.W. LEIBNIZ, Méditations. 11: "Quand tout ce qui entre dans une notion distincte est à son tour distinctement connu, ou bien quand l'analyse en est menée jusqu'au bout, la notion est adéquate. Je doute cependant que les hommes puissent en donner un seul exemple parfait; toutefois les notions des nombres s'en approchent beaucoup. Mais le plus souvent et surtout si l'analyse est très longue, nous n'embrassons pas toute la nature de la chose à la fois; nous substituons alors aux choses des signes dont, pour abréger, nous avons coutume d'omettre l'explication dans le travail actuel de la pensée, sachant ou croyant que cette explication est en notre possession." 
"canon" linguistique, la compréhension mutuelle n'aurait jamais lieu. On peut même constater qu'elle fait totalement défaut là où les interlocuteurs ne peuvent pas compter sur un tel arrière-fond. II ne faut pas seulement songer à l'incompréhension dans laquelle se trouvent plongés des individus qui parlent des langues différentes (encore que la recherche d'une langue commune, gestuelle s'il le faut, soit toujours possible ici). On pensera plutôt à la situation de celui qui essaie de lire un texte ancien dans une langue qu'il maîtrise, mais sans pouvoir la comprendre parce qu'il n'en partage pas le canon. L'incompréhension, que certains logiciens aimeraient attribuer à l'absence de définitions claires et distinctes, provient souvent de cette incompatibilité des canons. Ce sont les canons qui nous permettent de nous comprendre. La théologie le saura seulement plus que les autres savoirs.

La canonicité répondrait ainsi à l'incapacité pour une raison finie de rendre pleinement compte de ce qu'elle sait. C'est que la dérive infinie de la logique des signes trouve un écho dans la logique de la fondation et de l'argumentation. Ce qui vient fonder quelque chose est toujours quelque chose d'antérieur qui doit à son tour pouvoir être fondé. C'est ainsi que l'explication scientifique remonte de conditions en conditions dans ses entreprises de rationalité. Mais quand pouvons-nous nous arrêter au juste? Nulle part, en fait, mais l'angoisse toute cartésienne devant cette absence de fondations dernières aurait pour effet de paralyser les capacités pourtant réelles de notre savoir. D'où la nécessité - en science, en droit et dans tous les autres domaines où une justification publique est requise - d'en appeler à des autorités, à des précédents, à des études antérieures qui auraient fait le point sur une question. Toutes ces références exercent une fonction tacitement canonique: on peut s'appuyer sur cet ensemble ou ce niveau de savoir qui fait autorité. Le montrer en détail exigerait des ressources et du temps dont nul ne dispose réellement, vu l'impossibilité de couvrir tous les aspects d'une question dans l'espace restreint d'une seule vie. Variant une formule qu'avait employée autrefois Odo Marquard pour dépeindre la compétence des philosophes, on pourrait dire de la canonicité qu'elle est la compétence qui vient compenser notre incompétence 18 .

Le besoin d'un "canon" est particulièrement vital dans le domaine de la théologie. Qu'est-ce qui fait autorité dans un domaine - qui sait être celui de la foi - où personne, sauf quelques illuminés, n'est vraiment compétent? La raison se trouve ici confrontée à des questions qui la

18 Cf. O. MARQUARD, Abschied vom Prinzipiellen. Stuttgart, Reclam, 1981. 
dépassent. On pourrait prétendre que c'est parce qu'elles sont théologiques, relevant de l'invérifiable. C'est effectivement le cas, mais l'erreur serait de croire que la vérification ultime et la clarté logique parfaite fussent réalisées en d'autres provinces du savoir humain. C'est un dogme qu'a détruit l'épistémologie post-kuhnienne. Thomas Kuhn a montré, et la théologie aurait aussi pu lui servir d'exemple ici, à quel point des critères rhétoriques ou esthétiques étaient opérants dans les révolutions scientifiques. La canonisation de certaines doctrines, jugées plus acceptables ou plus élégantes que d'autres, vient utilement trancher un débat qui pourrait toujours se poursuivre. La raison théologique n'est donc pas la seule à s'occuper de questions qui la dépassent. Elle est seulement la seule à l'avouer.

L'exemple historique du canon des Écritures saintes peut être encore une fois éclairant. Il semble en effet que la canonisation de certains écrits ait souvent eu pour fonction de combattre des hérésies doctrinales en répondant à des situations de crise ou d'indécision. James Sanders a lumineusement rappelé que la canonisation de la Thora juive, qui fut le premier exemple historique de canonisation, avait pour fonction d'ancrer l'autorité du monothéisme chez un peuple qui restait continuellement tenté par le polythéisme et qui vivait le plus souvent dans un univers polythéiste ${ }^{19}$. La canonisation de certains écrits à tendance strictement monothéiste servait ainsi à établir la doctrine "vraie". La canonisation correspondait encore ici à une impuissance de la raison humaine. Comment en effet savoir si le monothéisme est plus vrai que le polythéisme? L'incompétence sur cette question, comme sur d'autres, est assez appréciable. La rationalité occidentale reconnaîtra sans doute dans le monothéisme son propre principe de l'économie des explications, mais cela ne rend pas le monothéisme plus vrai pour autant (un seul Dieu serait déjà beaucoup pour bien des scientifiques).

Notre propos n'est pas de gommer la différence de nature qu'il peut y avoir entre la théologie, qui est un savoir fondé sur une foi historique, et les autres sciences où les actes de foi sont peut-être seulement un peu moins manifestes. Nous cherchons uniquement à comprendre pourquoi la canonisation aura été appelée à jouer en théologie un rôle plus déterminant qu'ailleurs. La canonicité, la reconnaissance de l'autorité d'un texte ou d'une doctrine, apparaît moins nécessaire, par exemple, en mathématiques ou dans l'ordre des perceptions sensibles (où

19 Cf. James A. SANDERS, Canon and Community. A Guide to Canonical Criticism. (TUCKER, Gene M., ed.), Philadelphia, Fortress Press, 1984, 43. 
Jean Grondin: Canonicité et philosophie...

l'on peut espérer "voir" par soi-même). La canonicité théologique répondrait seulement à une ignorance plus criante, mais aussi plus honnête qu'ailleurs.

II y a bien sûr plusieurs autres différences entre le canon des Écritures pour la théologie et les réquisits canoniques des autres savoirs, voire du langage lui-même. Les Écritures saintes ont fait l'objet d'une canonisation expresse (quoiqu'elle paraisse avoir demandé plusieurs siècles avant d'avoir été définitivement arrêtée - au nom, bien sûr, des normes qui étaient alors canoniques). Le canon est devenu une institution dont on peut mettre en cause l'autorité, mais dont on ne modifiera pas la canonicité, ce qui n'est pas le cas des autres savoirs qui peuvent s'offrir le luxe de donner congé à des aberrations. Le canon des Écritures n'a pas cette latitude, même s'il peut être soumis à une pléiade d'interprétations. Mais cette clôture du canon ne trahit peut-être pas, encore une fois, un retard dogmatique de la théologie. - En fait, la clôture n'est elle-même que relative puisque l'ensemble du "canon" ne se limite pas aux seules Écritures saintes. L'horizon du canon comprend aussi les conciles, voire les encycliques, les lettres pastorales, les synodes, les grands projets théologiques, bref, toute les proclamations plus ou moins autoritaires, plus ou moins constantes de l'Église à travers les siècles. La pratique n'est plus au goût du jour, mais on parle aussi de "canonisation" dans le cas des saints, dont le modèle se trouve ainsi proposé à l'édification des croyants 20 . Bien sûr, l'autorité des saints et des conciles n'est pas celle des Écritures, mais elle contribue aussi à constituer un fonds de tradition et de mémoire qui permet à l'Église de se remettre continuellement en question. - C'est justement ce sens réfléchi du travail de l'histoire, de la mémoire, sinon de l'archive, qui distingue la canonicité proprement théologique. Les autres modes de canonicité que nous avons considérés, ceux de la science et, plus radicalement, celui du langage, ont une mémoire moins tenace.

20 En fait, il n'est pas si sûr que l'idéal de sainteté soit aussi démodé. Dans la culture moderne, les saints ont seulement été remplacés par d'autres modèles: les vedettes du cinéma, du sport et de la musique populaire, bien sûr, mais aussi les savants, les écrivains ou même les philosophes qu'il nous arrive d'idéaliser, et parfois d'imiter, lors même que nous nous efforçons de penser de façon autonome. D'autres érigeront des autels aux businessmen, aux prêtres ouvriers, etc. Puisqu'elles doivent bien partir de quelque part, l'existence et la pensée humaines se fondent toujours sur des modèles canoniques qui opèrent à des degrés divers comme de vagues exemples d'émulation ou de pureté. Encore une fois, l'Église, même si elle peut apparaître dérisoirement anachronique et idolâtre sur ce point, est peut-être la seule à le reconnaître pleinement. 
Mais cette canonicité "interne" n'est pas la seule à laquelle l'Église et la théologie soient exposées. La discours sur Dieu, ou de la foi, est aussi redevable des "canonicités" qui commandent les autres sciences et qui se sont succédées au fil des siècles. La théologie ne peut pas ne pas tenir compte de l'état actuel des sciences humaines et des exigences de son époque. Et il en a toujours été ainsi. C'est ainsi que les premiers chrétiens se sont inspirés de la philosophie grecque ambiante au même titre où les théologiens d'aujourd'hui peuvent prétendre parler au nom des acquis de la raison historique et critique. Les oeuvres de Copernic, Freud et Darwin, ou encore celles de Weber, Heidegger ou Habermas, jouissent en théologie de la même valeur "canonique" qui est la leur dans les savoirs où ils font autorité. La théologie ne saurait se refuser à aucune vérité. On pourrait dire que tout son défi consiste à équilibrer les canonicités qui traversent sa problématique essentielle. Elle doit, pour reprendre une formule célèbre de Gadamer, réussir une "fusion des horizons" 21 entre le canon traditionnel et la perspective de la foi dans un monde voué à la démythologisation. Gadamer voulait signifier par là que la compréhension devait satisfaire à la fois les exigences du présent et celles de l'objet passé qui commande tout son travail. II y a "fusion" parce que la distinction entre les deux horizons n'est jamais claire et nette. L'horizon du présent - disons celui de la théologie contemporaine est lui-même redevable du passé, de tout l'héritage de la théologie et des sciences occidentales, mais l'horizon du passé, celui du canon lui-même, ne peut pas être porté à la parole, même dans ce qu'il a de dépaysant et d'étranger, sans être plié aux capacités du discours d'aujourd'hui. L'un des privilèges de la théologie, redisons-le, est d'avoir une conscience expresse de la nécessité d'accomplir une telle fusion des horizons, du passé et du présent, du canon et des canonicités contemporaines. Elle est donc l'une des seules à pouvoir exercer un certain contrôle sur ses propres canonicités, d'autant plus d'ailleurs que le conflit entre le canon des Écritures et celui des évidences modernes est assumé comme tel. La théologie est ainsi en mesure d'accomplir de propos délibéré une fusion de ses horizons et de savoir ce qui lui arrive lorsqu'elle comprend.

La théologie est donc aussi soumise à la canonicité profane, mais, à la différence des autres sciences, son canon à elle reste immuable, dans sa textualité sinon dans sa signification. La théologie et l'Église possèdent un point de référence qui a la vertu de la constance. Mais on peut se demander, pour conclure, si ce canon fondamental relève encore du

21 Hans-Georg GADAMER, Wahrheit und Methode. Tübingen, Mohr, 1960; Vérité et méthode. Paris, Seuil, 1976, 147. 
Jean Grondin: Canonicité et philosophie...

canon lui-même, c'est-à-dire de l'ordre de la textualité? Autrement demandé: est-ce bien le texte fondateur qui fonde la religion chrétienne? Théologiquement, ce n'est pas sûr. En canonisant expressément une série de textes qui rendent témoignage de l'acte de salut qui a propulsé la religion chrétienne, ce qu'on aura voulu conserver, c'est en effet moins l'infaillible vérité de quelques textes, voire des récits eux-mêmes, dont la littéralité est toujours contestable, que le sens du témoignage de foi qui a été rendu, ou risqué. Les textes fondateurs de la chrétienté ne sont que des représentants d'une vérité qui n'est pas elle-même texte et qui transcende tous ses textes et leurs contextes. D'emblée, ces textes sont moins "canoniques" que l'événement dont ils ne voudront être que la trace. Ce serait un contresens - et dont les premiers auteurs auront peut-être eu eux-mêmes conscience - que de prendre tous ces récits au pied de la lettre. La canonicité, bien comprise, se relativise déjà ellemême. Elle s'ouvre et veut ouvrir à un événement de salut qui dépasse souverainement tout ce qu'on en pourrait énoncer. Par là, la canonicité renvoie à une autorité qui n'est pas humaine, mais qui ne se manifestera jamais. Ceci est également vrai des autres sciences où la vérité se trouve reportée à un progrès infini, mais aussi du langage puisque la présence ultime du sens y est toujours différée. Cela aussi, la théologie pourrait le savoir mieux que les autres. 